\title{
LA INMENSA VITRINA VIRTUAL EN LA SOCIEDAD DE CONSUMIDORES ${ }^{1}$
}

\author{
RAFAEL BACCA CONTRERAS ${ }^{2}$ / ELIZABETH GARCÍAMANTILLA ${ }^{3}$ \% \\ JOSÉ ALBERTO PINTO MANTILLA ${ }^{4}$
}

\section{RESUMEN}

El capitalismo contemporáneo, especialmente desde 1945, ha generado una sobre-excitación del deseo como principio rector. Los individuos han estado guiados por factores hedónicos, un creciente proceso de individualización de la sociedad, narcisismo, entre otros. Con todo, la sociedad consumista se ha ido estableciendo. Para analizar un aspecto de ello, se estudia cómo Internet se ha presentado -a través del comercio virtual -como una inagotable vitrina de mercancías. En este sentido, en un primer momento se aborda la transición de la sociedad de productores a la sociedad de consumidores. De otro lado, el texto se encauza en indagar el funcionamiento del marketing en Internet, en tanto que este es un hiper-potenciador del consumismo. Por ello, se indagan dos empresas principalmente: Amazon y Facebook; en aras de explicitar estas nuevas conductas y sus consecuencias. Por último, en el escrito se realiza un sucinto cuestionamiento sobre las repercusiones ecológicas de internet. En este orden de cosas, se pretende exponer el comercio virtual como una vertiginosa ventana al consumismo.

Palabras clave: consumismo, Internet, Facebook, Amazon y ecología.

\footnotetext{
${ }^{1}$ Este artículo fue presentado, en una versión preliminar, a modo de ponencia en el congreso "XI Jornadas de Sociología: Coordenadas contemporáneas de la sociología: tiempos, cuerpos y saberes", que se realizó los días 13 al 17 de Julio de 2015 en la Universidad de Buenos Aires, en la ciudad Buenos Aires, Argentina.

${ }^{2}$ Estudiante de noveno semestre en Economía Universidad Industrial de Santander. Cra 27 Calle 9 Ciudad Universitaria. Código Postal 680002, Bucaramanga, Colombia. Correo electrónico:

rafaelbacca08@gmail.com

${ }^{3}$ Estudiante de noveno semestre en Economía de la Universidad Industrial de Santander Cra 27

Calle 9 Ciudad Universitaria. Código Postal 680002, Bucaramanga, Colombia. Correo electrónico: eligarm@gmail.com.

${ }^{4}$ Doctor en Estudios Latinoamericanos de la Universidad Autónoma de México. Profesor en Economía de la Universidad Industrial de Santander. Cra 27 Calle 9 Ciudad Universitaria. Código Postal 680002, Bucaramanga, Colombia. Correo electrónico: jpinto@uis.edu.co
} 


\section{THE VAST VIRTUAL DISPLAY IN CONSUMER SOCIETY}

\section{ABSTRACT}

Contemporary capitalism, especially since 1945, has generated an overarousal of desire as a guiding principle. With this, on one hand the productivist system went from being led by the supply (producers) to be commanded by demand (consumers). And for another, individuals have been guided by hedonic factors, a growing process of individualization of society, narcissism, among others. However, the consumer society has been established. To analyze one aspect of it, you study how the Internet has been presented, through the virtually inexhaustible trade as a showcase of goods. In this sense, at first transition of society producer to consumer society is addressed. On the other hand, the text is channeled to investigate the performance of Internet marketing, while this is a hyper-consumerism enhancer. Therefore, two companies primarily investigate: Amazon and Facebook; in order to explain these new behaviors and their consequences. Finally, a brief written questioning on the environmental impact of internet is made. In this vein, it is intended to expose the virtual trade as a dizzying window consumerism.

Keywords: consumerism, Internet, Facebook, Amazon and ecology.

\section{Introducción}

La complacencia de un Mega Mall cuya característica capital sea que no cierre, que se mantenga despierto y a la orden, parece ser que solo lo ha podido conceder Internet. Los multitudinarios anuncios publicitarios, las incontables páginas alusivas a la(s) moda(s) y la siempre presente oferta de lo necesitado o lo que se está por necesitar, son algunos de los rasgos más sobresalientes de este medio como instrumento del consumismo. Cuyas implicaciones más directas son, entre otras, la satisfacción de siempre obtener lo apetecido en poco tiempo, sin movernos de nuestro lugar y la de mitigar cualquier angustia individual con las siempre a la mano- redes sociales o con la compra misma. 
En este texto se puede observar (además del fundamental cambio de una sociedad de productores a una de consumidores) el desarrollo y establecimiento de dos de los sitios más populosos y trascedentes en el universo de lo virtual: Facebook y Amazon. Esto permitirá apreciar más de cerca el pensar y actuar empresarial y comercial de estas famosas compañías, en aras de ratificar la suposición esencial del escrito: las redes sociales, y más evidentemente Amazon, son entes promotores de un consumismo rapaz e incesante. Los cibernautas pronto se encuentran y relacionan con infomerciales, pestañas-comerciales, y un largo etcétera que encarecidamente presagia y clama por una nueva adquisición.

Desde otra frontera, en la parte final, se presenta escuetamente la confrontación entre la forma ilimitada/artificial como se presenta la panoplia de mercancías, las consecuencias ecológicas (aunque no son alarmantes a primera vista) del uso de Internet. Igualmente, la propensión a certificar que en Internet son más los "pro" que los "contra" (limitando con mucho la posibilidad de crítica minuciosa) y cómo esta forma de existencia moderna repercute en la ecología del planeta.

2. Cambio de estación: sobre el paso de la «sociedad de productores» a la "sociedad de consumidores» ${ }^{5}$

El tránsito de un tipo de sociedad a otra es algo que estremece los cimientos mismos del pensar y hacer humanos. Por ende, no puede ser menos el análisis del mismo. Así entonces, el que se indague el paso de estar gobernados por un ideal del "hombre productivo", al cambio de bando del hombre que "experimenta lo nuevo/desconocido", que "no se sacia de consumir", no puede poseer una talla pequeña.

\footnotetext{
${ }^{5}$ Es ineludible aclarar que la idea de «sociedad de consumidores» no significa el hacer a un lado el aspecto productivo: que el individuo deje de trabajar para destinarse sólo a consumir, sería absurdo. Lo que se quiere indicar es la tendencia social. No sería posible afirmar que ha salido victoriosa una sociedad de consumismo cuando en la actualidad las horas de trabajo son más bien altas en comparación a tiempos pasados. En esta perspectiva, por ejemplo, se habla de "sociedad de prosumidores» para reflejar lo acontecido, no obstante acá se mantendrá la denominación de sociedad de consumo como tendencia social.
} 
En primera medida, la ubicación temporal inicialmente está entre la segunda mitad del siglo XIX y los albores del pasado siglo. Más aún, aquí es viable precisar lo que se entiende como «sociedad de productores». Este tipo de sociedad estaba caracterizada por un individuo con la bendición de Dios toda vez que trabajara incansablemente, debido a que mediante el trabajo agraciaba a Dios según Max Weber (2010). En este mismo sentido, este hombre poseía una dirección interna fuerte; era su fortaleza misma. Ello lo dotaba en creer que su autorrealización sólo dependía de su decisión, de sus fuerzas y mentalidad. En otras palabras, este hombre:

Está preocupado por sus propios asuntos y, por lo tanto, le preocupa perder tiempo; por otro lado, al no perder tiempo evita una autopreocupación ansiosa. Además, su relación con la gente, incluyendo a sus hijos, se da a través de su necesidad permanente y caracterológicamente condicionada de ponerse a prueba y disciplinarse. (Riesman, 1971, p.62)

Por lo que se puede notar el hombre de "dirección interna» (arquetipo de la sociedad de productores) era muy celoso con el tiempo; este era un tiempo traducido en necesidad.

El protestantismo calvinista, por su parte, lo permeó y su primer mandamiento era la famosa frase de Benjamín Franklin: "el tiempo es oro". La vertiginosidad del tiempo, en el que hombre debe descansar menos que las máquinas -que se fundían rápidamente por no dejarlas reposar -no permitía "botar" segundos con nadie, ni siquiera con un familiar. Esto, sumando a que su destino en la Tierra era trabajar para recompensar a Dios, dio como resultado una sociedad productivista. Cabe acotar de que en un primer momento la riqueza no era utilizada en su totalidad ni en un alto grado, puesto que se rechazaba el despreciable mundo terrenal y se ansiaba era salvar el alma para ir directo al Cielo; los resultados del trabajo sólo eran una prueba para agraciar a Dios. Sin embargo, y continuando con Weber, este espíritu netamente puritano mutaría para dar paso al disfrute de tan caudalosas riquezas.

En esta perspectiva, el individuo se caracteriza por procurarse una vida de riqueza, de acumulación material. Esto en aras de ser reconocido, de hacerse con 
fama. En suma: se esperaba escalar económicamente para liberarse de tener que trabajar por mano propia; el tiempo se quería dedicar a otra cosa, al ocio como fin en sí mismo. Así lo confirma Veblen (1974): "A los ojos de todos los hombres civilizados, la vida de ociosidad es bella y ennoblecedora en sí misma y en sus consecuencias" (p.46). El hombre de esta época sabía "lo que era la vida" y estaba presto en alma y cuerpo por conseguirla. Dicho de otro modo: "En esa época [sociedad de productores], un enorme volumen de posesiones sólidas, grandes, pesadas e inamovibles aseguraban un futuro promisorio y una inagotable fuente de confort, poder y estima personales" (Bauman, 2007, p.48). De igual forma, cabe señalar que "Pues no sólo el consumo, también la producción obedece en alto grado a procesos de ostentación" (Baudrillard, 2009, p.33). Ostentación que es fundamental en este tipo de sociedades (tanto de productores como de consumidores), a tal punto que el móvil rector es la emulación; como se evidencia en el libro "La teoría de la clase ociosa».

Sin embargo, como la producción estaba decidida intensamente por el "hombre de negocios" con la creencia de que "la oferta crea su propia demanda", así mismo, las mercancías estaban hechas con materiales durables. No existía el concepto de ser obsoletos, aunque sí, y esto ya conduce a un despropósito ecológico en tanto el tiempo de uso o el de vida útil es menor, se manipulaban las mercancías para que tuvieran menor calidad. En otras palabras, la idea antagónica para la ecología del planeta de la obsolescencia programa ya daba sus primeros pasos, especialmente en las mercancías alimentarias.

Análogamente, al fabricarse una mercancía se estaba construyendo, al mismo tiempo, la identidad de los trabajadores (García y Rodríguez, 2015). Una identidad duradera, pues el trabajo era "para toda la vida" y daba sentido a ésta; era un compromiso en el cual la persona no tenía elección. Así lo aclara Bauman (2005):

El tipo de trabajo teñía la totalidad de la vida; determinaba no solo los derechos y obligaciones relacionados directamente con el proceso laboral, sino también el estándar de vida, el esquema familiar, la actividad de relación y los entretenimientos, las normas de propiedad y la rutina diaria. (p.34) 
Identidad que mucho tenía que ver con el tipo de organización del trabajo y que terminaba definiendo la vida en general-, que luego iba a ser cronometradamente regulada por Taylor y seguidamente cimentada en la cadena de montaje Ford.

Ahora bien, en la «sociedad de consumidores» las cosas cambian radicalmente. En una primera instancia, es importante subrayar que el enfoque de dirección, en términos de Riesman, se canjea de ser interno a ser dirigidos por "los otros". Más exacto: "Así, el niño dirigido por los otros aprende en la escuela a ocupar su lugar en una sociedad en donde el grupo se preocupa menos por lo que produce que por sus relaciones grupales internas, su moral" (Riesman, 1971, p.88). O, lo que es lo mismo, el individuo se subyuga a la opinión pública. Es ahí, que radica el combustible de sus impulsos. Por ejemplo, la aceptación interiorizada de las modas populares en la vida de cualquier persona; o sino, es imaginar si alguien que tiene su moral propia y no quiere cambiar en poco tiempo de prenda, ¿cómo hacer para obligarlo? (a menos que la prenda ya no sea útil en un tiempo relativamente corto, siendo esta la función de la obsolescencia programada).

De esta manera, la construcción de la identidad de las personas se convierte en algo temporal, regido siempre por la feria de las vanidades; convirtiéndose en un bien de consumo más, pues según Bauman (2005): "Los caminos para llegar a la propia identidad, a ocupar un lugar en la sociedad humana y a vivir una vida que se reconozca como significativa exigen visitas diarias al mercado" (p.48). Así, la vida se configuró no ya en relación al trabajo sino a la capacidad de consumo, que en últimas es la capacidad de elección entre una u otra mercancía. El trabajo mismo o las muchas horas de trabajo son más bien un medio para alcanzar, luego de la remuneración, a deambular en los centros comerciales y arroparse con la identidad emergente.

El aspecto de la concepción del tiempo, por otro lado, varió radicalmente: "la idea del "tiempo de necesidad" ha sido reemplazada por el concepto de "tiempo de oportunidades, tiempo aleatorio, abierto en todo momento a la imprevisible 
irrupción de todo lo nuevo" (Bauman, 2007, p.54). Siguiendo este hilo, también se desculpabilizó utilizar el tiempo en otro aspecto, "Mientras los grandes almacenes contribuían a desculpabilizar el acto de comprar, el ir a tiendas, el «mirar escaparates» se convertía en una forma de ocupar el tiempo, un estilo de vida de las clases medias" (Lipovetsky, 2010, p.27). El tiempo ahora estaba destinado a los nuevos templos de la vida moderna, a consumir la mayor cantidad y rápidamente; esta será la nueva gracia divina (Sicerone, 2015). Y si bien no es posible participar activamente del mundo consumista en su espacio más preciado: el centro comercial; al menos, el individuo asiste a merodear las luminosas y vividas mercancías de las vitrinas, esto le aplaca momentáneamente el ansia de compra y le permite demostrar públicamente que todavía está vigente su tarjeta dorada de cliente elite, cédula de ciudadanía del mundo consumista.

La pensadora judío-americana, Hannah Arendt (2011), afirma que "La humanidad socializada es ese estado de la sociedad en el que sólo rige un interés, y el sujeto de dicho interés es la humanidad o las clases, pero nunca el hombre o los hombres" (p.338). Contemporáneamente el interés único y que parece inamovible es el consumismo. Así entonces, no sólo los individuos cambian su sentido de vida, sino también los productores viran su móvil de producción en lo que opina el público, en lo que dicen "los otros": el lema de la sociedad consumista es "el consumidor siempre tiene la razón". No obstante, es crucial atemperar esta exclamación. Los monopolios, en contra de todos los mandamientos neoliberales, ejercen su gran poder para determinar lo que saldrá o no al mercado (Blacha, 2014); más bien lo que expresa la frase es que -gracias al Internet por ejemplo- los individuos pueden ejercer más públicamente sus gustos y ello lleva a que se refleje en los novedosos productos 0 , en muchos casos, rechacen de tajo un producto nuevo sin importar que se venere a la marca.

Por último, vale decir que los productos ya no se acercan a exprimirlos hasta que den su última gota, ahora su utilización es más leve, de momento. Esto lo indica el momento-moda. De igual modo, factores como la publicidad, la obsolescencia programada y simbólica y el crédito han aumentado 
considerablemente desde mitad del siglo XX. Estos instrumentos han ocasionado graves daños a la ecología del planeta, a tal punto que algunas investigaciones informan que serían necesario tres planetas tierra para sostener un estilo de vida consumista generalizado; este aspecto es tratado al final pero relacionado solamente con Internet. La funcionalidad de estos instrumentos, pues, es esencial para la cultura consumista; no obstante, el texto se concentra en estudiar sólo uno de esos factores: la publicidad. Siendo este elemento un movilizador del comercio virtual y de gran beneficio para las empresas de servicios que incursionan en estos nuevos procesos de compra y venta acelerados.

\section{Dos estanterías virtuales en el fascinante mundo de la mercancía}

El «arsenal de mercancías» tiene uno de sus más grandes florecimientos en el Internet: a tal punto que, "fácilmente, es el mercado y escenario social más grande del mundo" (Pinto, 2013, p.354). Esta proliferación y accesibilidad sencilla a tantos objetos, nos presenta la posibilidad de percibir -aparentemente- una ilimitada serie de mercancías; no nos deja sombra de duda de que podemos tener lo deseado aquí y ahora. El papel, pues, del denominado «comercio virtual» tuvo su "Big Bang" y sus tentáculos están abarcando cada vez más empresas e individuos. Para poder comprender mejor esta nueva arista del proceso comercial, especialmente desde el ámbito de la publicidad ${ }^{6}$, en Occidente, seguidamente se exponen dos reconocidas empresas: Facebook y Amazon.

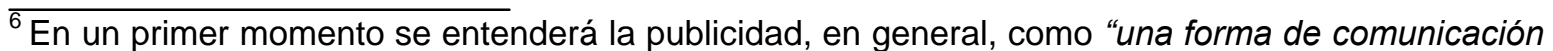
masiva cuya finalidad es transmitir información y/o incidir sobre las actitudes (creándolas, modificándolas o reforzándolas) para impulsar a los destinatarios de la misma a un comportamiento favorable a los intereses del anunciante." (Prat, 2005). Sin embargo, no sobra decir que sobre la publicidad existe un extenso debate: desde posturas que expresan que su uso es persuasivo, manipulador, hasta que es un excelente instrumento para la democracia, en tanto nos tiene al corriente de lo nuevo. Por último, en el texto mismo se concluirá que ni la publicidad es totalmente ingenua ni exclusivamente orwelliana, pero sí tiene matices en que se inclina por una u otra.
} 


\subsection{Facebook}

La evolución de Facebook ha estado marcada por su acelerado crecimiento empresarial. Este oscila entre el vertiginoso número de usuarios, las diversas, y a veces fracasadas, aplicaciones y los nuevos tentáculos financieros. Es necesario ir por partes. La idea de esta red social germinó en la universidad de Harvard, de la mano de Mark Zuckerberg. En un primer momento, Mark lanzó en 2003 -a escala universitaria- Facemash, cuya finalidad era compartir/comparar fotografías y nombres de estudiantes. Ello es bastante familiar en Estados Unidos: "Los "facebooks" son publicaciones que hacen las universidades al comienzo del año académico, que contienen las fotografías y nombres de todos los estudiantes y que tienen como objetivo ayudar a los estudiantes a conocerse mutuamente" (Cad, s.f.). Poco tiempo después, el estudiante de Harvard presenta thefacebook; la cual ha sido la base de una fuerte polémica, puesto que según han denunciado los hermanos Winklevoss y Divya Narendra es una copia de su idea denominada HarvardConnection.com ${ }^{7}$.

Para el 2004 ya Facebook toma forma: "Zuckerberg lanzó la primera versión de la red social. Rápidamente se convirtió en uno de los sitios predilectos para los estudiantes de Harvard" (Infotechnology, 2014). Debido a la acogida en la universidad norteamericana, varias universidades se fueron sumando a la red ${ }^{8}$ : "MIT, Boston University y Boston College y las más prestigiosas instituciones de Estados Unidos" (Cad, s.f.). Seguidamente, el imperio virtual de Zuckerberg amplió su radio de acción al traducir, entre el año 2007 y 2008, al español a Facebook hoy funciona en más de 70 idiomas. En este sentido, la empresa para el año 2007 ya contaba con 19 millones de usuarios y en la actualidad se contabilizan en más de 1.300 millones.

\footnotetext{
${ }^{7}$ La discusión y el desarrollo de la disputa (al igual que la conformación de Facebook) es recomendable contextualizarla con la película The Social Network. En la misma es posible esclarecer un poco de la vida y personalidad del chico de Harvard.

${ }^{8}$ Vale acotar que la idea de "red social" en la virtualidad no es una categoría de análisis novedosa, ya que "La idea de crear una comunidad basada en la Web en que la gente compartiera sus gustos y sentimientos no es nueva, pues David Bohnett, creador de Geocities, la había incubado a fines de los años 1980" (tuteformas, 2011).
} 
Ahora bien, entrando en materia de las herramientas de Facebook en función de la publicidad (esta herramienta ha sido una de sus principales fortalezas desde sus albores) para estimular el consumo en sus cibernautas, la compañía creó F-commerce. Esto es, "Gracias a la unión del Social Commerce (comercio social) y del e-commerce (comercio eléctrónico), nace el f-Commerce, o comercio electrónico basado en Facebook" (Palbin, 2014). Este novedoso mecanismo comercial virtual tiene como ejes principales: exclusividad para el cliente, usabilidad y practicidad de compra, una oferta personalizada, inmediatez y contacto social (compartir lo que han comprado mediante su publicación principalmente). Con estos principios Facebook busca, por un lado, que las empresas o individuos "emprendedores" tengan su espacio comercial en un ámbito tan lúdico como esta red social, y, por otro, que los usuarios tengan la posibilidad de recibir información publicitaria diversa y en grandes cantidades. Hace algunos años un estudio realizado por Hanvas Media y Social Lightspeed precisaba que:

El estudio encontró que mientras que la industria está aclamando el comercio social como la próxima gran revolución, parece que aún no han visto que el $89 \%$ de las personas no ha comprado nada a través de Facebook y lo que es peor, el 44\% aún no está interesado en hacerlo. (Álvarez, 2013)

Sin embargo, investigaciones más recientes dan la razón del posible éxito del F-commerce en el sentido publicitario: "Más de 30 millones de pequeñas y medianas empresas de todo el mundo utilizan Facebook." Esto suma "1,5 millones de anunciantes activos de julio de 2014." A la par de esto, los nuevos dispositivos que reciben la publicidad han aumentado, "La porción de publicidad en dispositivos móviles aumentó a $69 \%$ del total en el último trimestre, contra $66 \%$ los tres meses anteriores" (Semana, 2015). En últimas, la eficacia de esta herramienta es todavía muy dispar, no obstante su crecimiento es alto.

La empresa del joven multimillonario norteamericano, con puesto en la distinguida revista Forbes, no solo se ha dedicado a formar nuevos espacios en "la nube" para que las empresas puedan publicitar, sino que ha realizado experimentos clandestinos para observar la reacción de los usuarios. En tal 
perspectiva, un «experimento psicológico» se realizó en 2007: "El ensayo, de dudosa aceptación social, consistió en manipular el algoritmo que selecciona las noticias que aparecen en el muro de cara usuario con el objeto de observar si existe un «contagio emocional» entre las personas" (Sánchez, 2014). Concluyendo que "la comunicación textual tiene un impacto determinante en la transmisión de emociones" (Sánchez, 2014). No sería extraño que estos "experimentos" tuvieran tanto, fines en campañas políticas como de marketing. El control de datos tan transcendentales en las personas por parte de Facebook, saluda equiparablemente al del buscador más importante en la red: Google.

Cabe acotar que el algoritmo empleado actualmente por Facebook (Open Graph) "podrá conectar gustos y opiniones de millones personas y gestar un APIalgoritmo de búsqueda que va un paso más allá del desarrollado en Google". Por lo que, "se podrá personalizar totalmente una tienda basándose en tus compras anteriores, gustos, preferencias de tus amigos, etc..." (Chulilla, 2010). Será bastante sencillo para empresas de un lado saber qué quieren los consumidores y de otro qué aceptarían en el mercado. Le es más fácil, pues, a alguien confesar en Facebook -ya se a través de un me gusta, comentario, publicación, etc.- sus gustos que decirlos en público. Y de cierta forma ahí radica la importancia de estos sitios para recolectar muy receptivamente las inclinaciones de las personas y venderlas al mejor postor.

Por último, es ineludible ocultar las excesivas ganancias a través de los años de esta compañía. A tal punto que es la única compañía siendo "red social" en la lista (tomado del sitio web The Atlantic) de las cincuenta empresas que poseen más utilidades en Internet. Actualmente "El gigante mundial anunció en enero que su beneficio anual casi se duplicó en 2014, situándose en 2.900 millones de dólares, y que tanto el número de usuarios como los ingresos por publicidad móvil siguieron aumentando" (Semana, 2015). 


\subsection{Amazon}

Desde la puesta en marcha de Internet y el establecimiento de sus siglas "WWW" (World Wide Web), las empresas en todo el mundo empezaron a cambiar sus estrategias de venta y publicidad. Una de las empresas pioneras en apostar al negocio de Internet fue "Cadabra.com" fundada por Jeff Bezos en 1995. Una empresa diseñada para la búsqueda fácil de libros en la red, la cual -por una estrategia de marketing- se convertiría en lo que hoy en día se conoce como Amazon, con el fin de figurar en los primeros puestos de búsqueda dado el ordenamiento alfabético de los sitios web.

Al desmitificarse las compras por Internet, Amazon logró revolucionar el comercio electrónico debido al seguimiento que le permite hacer al usuario de todo el proceso de compra y de pos-compra. Para este tipo de empresas que tiene una oferta infinita ${ }^{9}$ y una economía de escala, en la que priman los costes variables sobre los costes fijos, es fundamental la innovación tecnológica. Por ello, Jeff Bezos, en el 2013, anunció una nueva forma de distribución de la mercancía que renovaría las compras online. La meta es realizar, en primera instancia, pequeños envíos domiciliarios con drones; así el consumidor podrá adquirir el producto en un lapso, aproximadamente, de media hora. Sin embargo, el comercio virtual debe afrontar el problema del envío de mercancía a lugares remotos, ya que "en estas décadas de Internet se ha mejorado mucho en la tecnología, desde las webs a los procesos de compra, pero el transporte de la mercancía ha permanecido inalterado" (Pozzi, 2013). Por ello, la compañía de Bezos advierte que dentro de cinco años se debe avanzar en el transporte aéreo para envíos de paquetes personalizados.

No obstante, este tipo de tecnologías apuntalan en la sociedad civil el frenesí por la inmediatez y la velocidad de consumo. Esta empresa ha cambiado radicalmente la forma de comprar y vender productos, puesto que ya no es necesario el desplazamiento del cliente a la respectiva tienda sino que basta sólo

\footnotetext{
${ }^{9}$ Es necesario aclarar que al referirse a "una oferta infinita", se debe entender como las promesas que venden este tipo de empresas según su publicidad.
} 
con un click para tenerlo a la mano. Amazon se posiciona como el gigante del comercio electrónico, porque ha hecho que muchas empresas vendan sus productos por medio de esta plataforma; así pueden diversificar sus potenciales consumidores pues no están restringidos al stock de una pequeña tienda, sino que cuenta con un catálogo infinito.

Este tipo de beneficios que ofrece las llamadas "burbujas empresariales" han obligado a las demás empresas a buscar otros medios de publicidad, así como, bajar sus precios debido a las presiones que ejercen este portal de servicios sobre los proveedores. Ya no basta, pues, una buena publicidad para persuadir, sino que también la calidad tanto del producto que se publicita como de la eficiencia en el transporte es crucial para el comercio virtual; cabe destacar el algoritmo actual de Google se basa en la calidad del producto, de la publicidad y el precio para posicionar a la empresa en el momento de responder a una búsqueda. Esto de cierta manera atornilla a los monopolios como el caso, en algunos productos, de Amazon, puesto que es bastante complicado escalar, posicionarse y combatir en calidad y precios con este tipo de empresas. Más aún cuando estas lanzan sus propias plataformas para reproducir el contenido de sus ventas: Kindle de Amazon o Apple es un ejemplo paradigmático, entre otros.

Muchos periódicos y portales de noticias han clasificado la estrategia comercial de Amazon como agresiva. Por ejemplo, en el 2014:

El grupo de comercio electrónico, que vende dos de cada cinco libros en EEUU, quiere que se rebajen los precios de los ebooks. Y, para presionar a la editorial francesa a que cumpla sus deseos, ha retrasado la entrega de algunos títulos y bloqueado la compra de otros tantos. (Bond \& Mance, 2014)

Esta batalla por mantener precios bajos para los clientes mientras se ponen en riesgo las editoriales y las librerías en la era digital, no es más que la muestra del control que tiene Amazon sobre las compras en línea.

Lo que alguna vez se tildó en los medios de comunicación como una estrategia de "democratización de las compras" -más personas con mayor acceso a productos internacionales-, ahora es una estrategia agresiva para absorber las ventas de las tiendas, almacenes y empresas a través de su portal de servicios. 
Así, aspectos cotidianos de la vida como la lectura, las compras y la diversión se han virtualizado. Aspectos mayoritariamente públicos como la diversión y las compras, pues, cambian de terreno a uno más privado, más íntimo y por tanto personalizado. Esto es visible en empresas como Nexflix que permiten escoger de forma especializada una película dadas sus opciones de filtrado, y con estos datos luego recomendar largometrajes relacionados a los gustos del consumidor.

Es evidente que el comercio electrónico ha traspasado fronteras económicas y sociales. Se ha glorificado como la herramienta que "democratiza el consumo" sin importar las condiciones internas de cada país, puesto que los portales de servicio nunca cierran sus puertas y, según la innovación del transporte de mercancías por medio de drones, su alcance es global. De ahí, que un portal como el Espectador Digital haya analizado el modelo de negocio de este tipo de empresas, en las que la «teoría de la larga cola» es la base de la economía de internet. Pues "se traduce en que ya no es necesario tener la oferta de un mercado restringida por su capacidad de almacenamiento" (Archanco, 2013). Así, como lo plantea el artículo: "Una librería está limitada por la cantidad de libros que puede tener expuestos en sus estanterías [...] La librería se concentra en vender pocos libros pero en grandes cantidades, dejando fuera un gran número de títulos de nicho" (Archanco, 2013).

En cambio, un portal como Amazon vende un puñado de millones de títulos diferentes de libros, lo que equivale a mayores ganancias en vez de vender gran cantidad de una pequeña selección de libros. Cabe añadir que la idea de ilimitado distorsiona la percepción de los consumidores porque observan en la virtualidad un universo de mercancías que se regeneran y que pareciera que no tuviera coste alguno o fuese precario en términos ecológicos. $O$, así mismo, se publicita que la compra de ese producto ayuda a paliar el cambio climático y con ello sacia el pecado de la "huella ambiental" de su compra.

Sin embargo, el almacenamiento que tiene esta empresa le genera costes fijos al igual que manejar una distribución tan grande; pero esto no funcionaría sino hiciera millones de pedidos. No en vano, el director de Amazon sacrificó las 
ganancias de finales del 2012 y del 2013 para elevar las ventas, las cuales pasaron de 48.077 millones de dólares en el 2011 a 88.988 en el 2014, según el estado de resultados de la empresa (Portafolio, 2014). Un movimiento comercial que le generó a la empresa en el primer trimestre del 2014 beneficios netos de 108 millones de dólares, "un 32\% más que el mismo periodo del 2013" (Portafolio, 2014).

Así entonces, la lógica de empresas como esta busca conocer los gustos y aversiones de sus clientes a toda costa, sin prestarle mucha atención a las necesidades de sus propios trabajadores. Por lo que en el tercer congreso mundial de la Confederación Sindical Internacional (CSI) celebrado en Berlín en el 2014, Jeff Bezos fue ganador del peor jefe del mundo, pues Amazon trata a sus trabajadores como máquinas. Según la Secretaria general de la CSI, "La empresa no esconde que en tan solo unos pocos años les sustituirá por robots. Se trata de una rica empresa estadounidense que opera a escala mundial despreciando la dignidad y los derechos de la gente trabajadora" (Internacional, 2014). Esto deja en evidencia el direccionamiento del sistema de servicios, al igual que sus intereses; así como la manifestación más concreta de una sociedad de consumo.

\section{Panorama ecológico}

Ahora bien, las repercusiones ecológicas negativas que se desprenden del uso de Internet en la vida cotidiana no son tan profundas como las de otras prácticas. Concretamente se observa que:

Abrir una página web supone la emisión de 20 miligramos de dióxido de carbono (CO2), el principal gas de efecto invernadero (GEI) implicado en el cambio climático, según la página web de General Electrics "Data Visualization". Cada mes internautas de todo el mundo están 35.000 millones de minutos online, según datos de 2012 de la compañía GoGulf.com. (Muerza, 2013)

De otro lado,

La electricidad utilizada para transmitir los trillones de mensajes no deseados (spam) durante un año serviría para más de dos millones de hogares, según la empresa de antivirus McAfeee. Las emisiones de $\mathrm{CO} 2$ equivalen a las producidas por tres millones de coches". De igual forma, la industria global de las nuevas tecnologías genera una cantidad de GEI 
similar a los de todas las compañías aéreas del mundo, según la consultora Gartner. (Muerza, 2013)

No obstante, si bien las incidencias que deterioran o aceleran el recalentamiento del planeta por el uso de la red no son para intranquilizarse sobremanera, sí lo es lo que se ha denominado el "fetichismo tecnológico". Este fetichismo incentiva el adormecimiento del interrogante sobre en qué se sostiene Internet, cuáles son sus acontecimientos tanto en el hecho mismo de usarlo como en el cyber-relacionamiento con el mundo. Por ende, este soporífero tecnológico muchas veces le gana terreno a la crítica, dando por hecho que "son más las cosas buenas".

Los discursos de la globalización sobre los beneficios al "interconectar" diferentes culturas, han llevado a que no se pueda pensar un mundo sin Internet, sin tecnología, sin tecnociencia. Como afirma Cornelius Castoriadis (2008): "La dificultad del hombre moderno para admitir la eventual nocividad de la tecnociencia no deja de tener una analogía con el sentimiento de absurdo que experimentaría el fiel ante la aserción: Dios es malo" (p. 71). Se obvia el límite de la biosfera sin siquiera considerar que no es tan sólo el daño ambiental que produce entrar a una página web. Este tipo de sitios reproducen un estilo de vida consumista -dado la inmensa publicidad encontrada en ella, por ejemplo- que requiere de una producción fija y sin descanso; lo cual no es otra cosa que contaminación y explotación de la naturaleza. No obstante, estos problemas se mitigan o tienen poca relevancia en la mayoría de individuos si se les compara con otros beneficios de la red y de la tecnociencia.

\section{Consideraciones finales: Un llamado de atención}

El cambio de estación presentado en el texto, argumenta de forma meridiana que la voluntad del individuo parece haberse trasteado: el impulso que dictamina qué hacer, es otro. Ya no será el hombre con afán de crear nuevos inventos, de provocar ingentes cantidades de objetos, de ser un gran productor el que defina a la sociedad occidental. Sino más bien un individuo más socializado, dirigido por el peso de las palabrerías de las masas (por ejemplo, lo que impone el 
grito de la última moda), en últimas, un individuo centrado en el consumo. El consumo será, entonces, la quintaesencia de la existencia.

Con un terreno ya abonado en cuanto al ser-en-el-mundo, el Internet, luego de su primera forma (como instrumento militar), aparece para suplir las desventuras humanas: "El servicio dejó de ser sólo el aporte al área militar y científica, para satisfacer las urgencias cotidianas de una vida solitaria y a veces sin sentido" (Pinto, 2013, p.354). Este medio, a través de redes sociales como Facebook o Twtitter, y de empresas colosales como Amazon, ha satisfecho a más de una persona no sólo en su búsqueda de información o comunicarse con un contacto, sino también para realizar compras que pudieron 0 no estar programadas. La publicidad, entonces, protagoniza un papel esencial para seducir al siempre potencial comprador que es toda persona.

Ahora bien, la publicidad es fundamental debido a que se debe saber el contenido y los efectos de la mercancía, entre otros datos relativos a ella, lo que en cierta forma confirman compañías como las expuestas acá es que este instrumento no sólo funciona para informar. En tanto ya se realizan experimentos en los que se "conoce" al futuro cliente, esta forma de proceder ya no sólo debe contenerse en un neutral instrumento sino en el campo de la manipulación del consumidor. Por ende, la publicidad es neutral en tanto se utilice para informar, pero los grandes monopolios parecen suponer que los alcances de la misma deben ir más allá de eso.

Si bien el e-commerce no ha cumplido con las expectativas de años anteriores, es muy prematuro formular su fracaso. Igualmente, podemos apreciar que las dos empresas presentadas han escalado en estimular la publicidad en el mundo virtual. Si para Amazon es sentido común hacerlo, dado que es un gran centro comercial virtual, para Facebook no, pero aun así la publicidad ha conseguido la propiedad de la ubicuidad en esta red social. Por ende, uno de los tentáculos más importantes de consumismo abre un mundo de nuevos mercados; ahora en el individuo tendrá un gran peso ser tele-consumidor. 
Por último, es notable que la publicidad nos expone un mundo sin complicaciones, sin diatribas, además de un planeta con recursos infinitos. El que un objeto se renueve, o prometa hacerlo en pocos segundos, horas o días, es muestra tangible de lo desmesurado con que la fluctuación entre oferta y demanda se dinamiza en la vida virtual. Las repercusiones de esta manera de percibir el planeta desbordan con mucho las pretensiones de nuestro escrito, pero la alerta y problematización de esto no la podemos pasar por alto.

\section{Referencias Bibliográficas}

Álvarez, M. A. (2013). puromarketing. Obtenido de http://www.puromarketing.com/76/12916/commerce-comercio-electronicofacebookresultado-fracaso.html

Archanco, E. (10 de Junio de 2013). El Espectador Digital. Recuperado el 25 de Febrero de 2015, de http://elespectadordigital.com/el-modelo-de-negocio-deamazon-es-la-adiccion-al-riesgo/

Arendt, H. (2011). La condición humana. Barcelona: Paidos .

Baudrillard, J. (2009). La sociedad de consumo: Sus mitos, sus estructuras.

Madrid: Siglo XXI editores.

Bauman, Z. (2005). Trabajo, consumismo y nuevos pobres . Barcelona: GEDISA.

Bauman, Z. (2007). Vida de consumo. México: FCE.

Blacha, L. (2014). El orden social en perspectiva sociológica. Collectivus, Revista de Ciencias Sociales, Vol. 1, Núm.1, 4-27.

Bond, S., \& Mance, H. (24 de Agosto de 2014). Expansión. Recuperado el 26 de Febrero de 2015, de http://www.expansion.com/2014/08/24/empresas/tecnologia/1408904766.html cad. (s.f.). cad. Obtenido de http://www.cad.com.mx/historia_de_facebook.htm Castoriadis, C. (2008). El mundo fragmentado. La Plata: Terramar. Chulilla, F. (9 de Mayo de 2010). dinaweb. Obtenido de http://www.dinaweb.net/marketing/opengraph-facebook-revolucion-ecommerce/ infotechnology. (3 de Febrero de 2014). infotechnology. Obtenido de http://www.infotechnology.com/internet/La-historia-de-Facebook-paso-a-paso201402030003.html García, J. y Rodríguez, P. (2015). La identidad y lo popular: la identidad popular en tiempos de socialismo. Collectivus, Revista de Ciencias Sociales, Vol. 2, Núm.1, 439.

Internacional, C. S. (24 de Mayo de 2014). Confederación Sindical Internacional. Recuperado el 28 de Febrero de 2015, de http://www.ituc-csi.org/jeff-bezos-de- 
amazon-gana-el?lang=es

Lipovetsky, G. (2010). La felicidad paradojica : ensayo sobre la sociedad de hiperconsumo. Barcelona: Anagrama .

Muerza, A. F. (17 de Enero de 2013). consumer. Obtenido de http://www.consumer.es/web/es/medio_ambiente/energia_y_ciencia/2013/01/17/21 5388.php

Palbin. (29 de Septiembre de 2014). Palbin. Disponible en: https://www.palbin.com/customer/portal/articles/1507489-\%C2\%BFqu\%C3\%A9es-elfcommerce-

Pinto, A. (2013). Encuentro, destruccion y ocultamiento de las indias occidentales: Crónicas del pueblo indio y de otras culturas. Bucaramanga: UIS.

Portafolio. (24 de Abril de 2014). Portafolio.co. Recuperado el 27 de Febrero de 2015, de http://www.portafolio.co/negocios/ganancias-amazon-2014 Pozzi, S. (2 de Diciembre de 2013). El País. Recuperado el 25 de Febrero de 2015, de

Teconología: http://tecnologia.elpais.com/tecnologia/2013/12/02/actualidad/1385969482 968727 .html

Prat, J. T. (2005). Consumo, luego existo: Poder, mercado y publicidad. Madrid: Icária.

Riesman, D. (1971). Muchedumbre solitaria. Buenos Aires: Paidos.

Sánchez, J. (1 de Julio de 2014). ABC. Disponible en: http://www.abc.es/tecnologia/redes/20140630/abcifacebook-manipula-algoritmonoticias-positivas-negativas-estudio-psicologico201406300950.html

Sicerone, D. (2015). El marxismo des-colonizado como detracción a la crítica de Popper al historicismo. Collectivus, Revista de Ciencias Sociales, Vol. 2, Núm.2, 134-160.

Revista Semana. (24 de Febrero de 2015). Revista Semana. Disponible en: http://www.semana.com/tecnologia/articulo/facebook-pelea-por-la-publicidadeninternet/418989-3

Tuteformas. (17 de Junio de 2011). Tuteformas. Obtenido de https://tuteformas.wordpress.com/2011/06/17/\%C2\%BFfacebook Veblen, T. (1974). Teoría de la clase ociosa. México: FCE. Weber, M. (2010). La ética protestante y el espíritu del capitalismo. Madrid: Alianza Editorial.

\section{Para citar este artículo:}

Bacca, R., García, E., y Pinto, J. (2016). La inmensa vitrina virtual en la sociedad de consumidores. Collectivus, Revista de Ciencias Sociales, 3(1), 91-109. doi: http://dx.doi.org/10.15648/Coll.1.2016.5 